\title{
Surface Plasmon Modes of Dielectric-Metal-Dielectric Waveguides and Applications
}

\author{
Shivani Sital ${ }^{1}$, EnakshiKhular Sharma ${ }^{2}$ \\ ${ }^{1,2}$ (Department of Electronic Science, University of Delhi South Campus, India)
}

\begin{abstract}
The dielectric-metal-dielectric plasmonic waveguide structures find applications in integrated optics and fiber polarizers and sensors. Surface plasmon waves guided by thin metal film have been intensively studied over the last two decades. However, most studies have been confined to relatively low index dielectrics. With growing interest in silicon photonics and other semiconductors dielectric of relatively higher dielectric constant we carried out a detailed study of the modes supported by a metal filmbetween dielectrics of relatively higher dielectric constant. The study clearly shows that both modes, the "antisymmetric" $\left(a_{b}\right)$ short range and "symmetric" $\left(s_{b}\right)$ long range bound modes can exist only when the contrast between the indices is low. For high contrast the symmetric mode transforms into a leaky mode.For completeness we also includethe antisymmetric leaky $\left(a_{l}\right)$ mode and symmetric leaky $\left(s_{l}\right)$ mode in our study, although they are not important for guided wave structures. We have also included "leaky modes" in the bound mode domain as solution of the boundary value problem as reported in some early studies. We have also considered some applications of the DMD waveguides with an emphasis on identifying the participating mode in each application.
\end{abstract}

Keywords: Surface plasmon modes, dielectric-metal-dielectric waveguide, bound modes, leaky modes, sensors

\section{INTRODUCTION}

It is well known that the interface of the medium with a real negative dielectric constant and positive dielectric constant can support a guided surface TM mode. In a typical metal, the dielectric constant can be written as $\varepsilon_{m}=-\varepsilon_{m r}-j \varepsilon_{m i}$ [1,2], i.e., in addition to negative real part there is an imaginary part in the dielectric constant which manifests itself as loss. Hence, a semi-infinite metal dielectric interface [shown in Fig.1(a)] supports a lossy TM surface wave also known as a surface plasmon wave. Similarly, a thick metal slab with semi-infinite dielectric on either side [as shown in Fig.1(b)] can support such surface plasmon modes on either interface. However, for thin metal films these modes get coupled and the film can support one or two lossy bound surface plasmon TM modes depending on the metal thickness and dielectric constants of the two dielectric media.

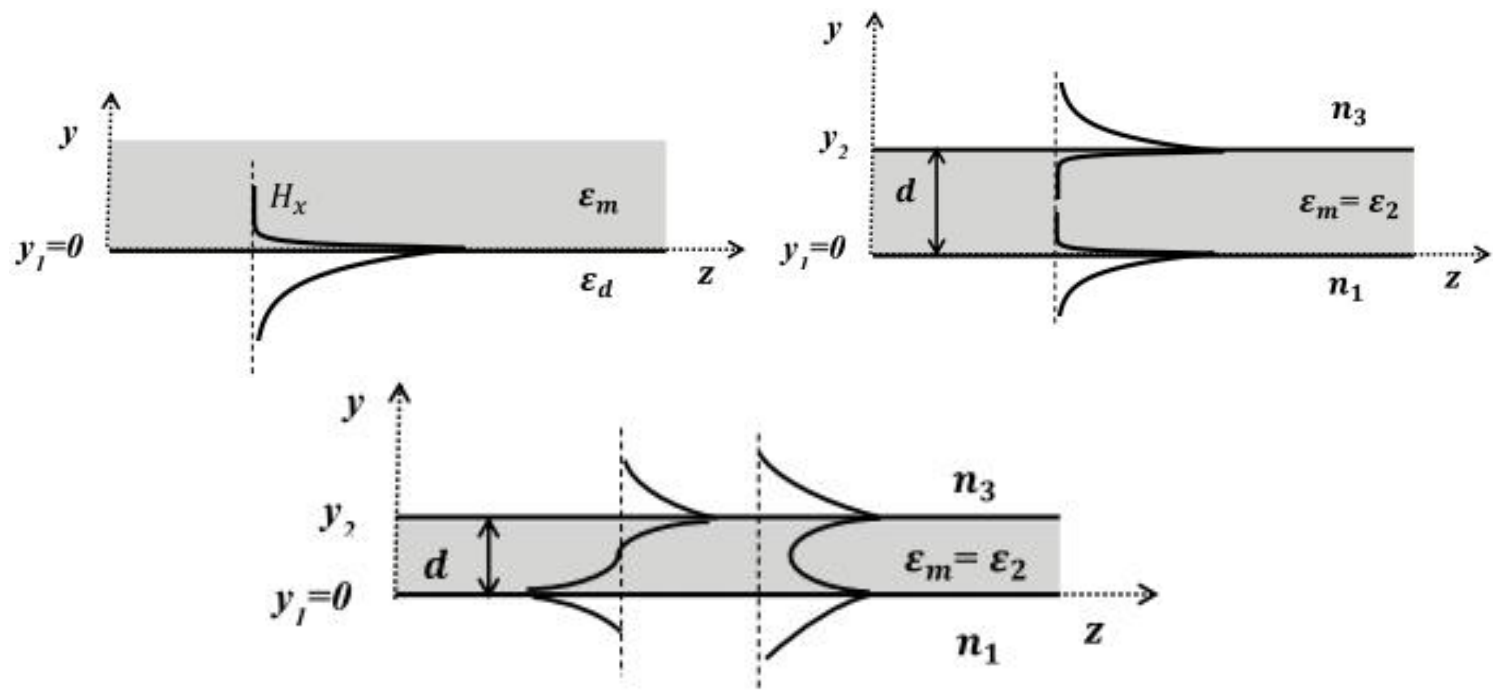

Fig.1 (a) Surface TM mode supported by a metal-dielectric interface. Surface modes supported by the dielectric-metal-dielectric (DMD) waveguide on (b) a thick metal slab, c) thin metal film.

Such dielectric-metal-dielectricplasmonic waveguide structures find applications in integrated optic and fiber polarizers [3-7] and sensors [8]. Surface plasmon waves guided by thin metal films on the dielectrics have been intensively studied over the last two decades $[9,10]$. However, most studies have been confined to relatively low index dielectrics. With growing interest in silicon photonics and other semiconductor dielectrics of relatively 
higher dielectric constant we carried out a detailed study of the modes supported by a metal film between dielectrics of relatively high dielectric constant. The study clearly shows that both modes, i.e. the "antisymmetric" short range and "symmetric" long range bound modes can be supported only when the contrast between the dielectric constants indices is low. At higher contrast, the symmetric bound mode changes into a leaky mode. Further, it is only in the low contrastregion that attenuation and effective indices of the modes vary significantly by change of metal film thickness. Some early studies on such thin metal film plasmonic waveguides have also included "leaky modes" in the bound mode domain as a solution of the boundary value problem [9,10]. Such modes are however not of any practical significance. In this paper, we will discuss in detail all the possible modes supported by the dielectric-metal-dielectric (DMD) waveguide and various applications of these modes in integrated optic devices and sensors.

\section{ANALYSIS}

The dielectric media on either side of the metal in plasmonic waveguides can in general consists of layers of different dielectrics in some applications. Hence, to study the characteristics of the TM modes supported by DMD plasmonic waveguide we have used the method developed for a multilayer planar waveguide of $\mathrm{N}$ layers (Fig.2) with each layer of complex refractive index or dielectric constant.

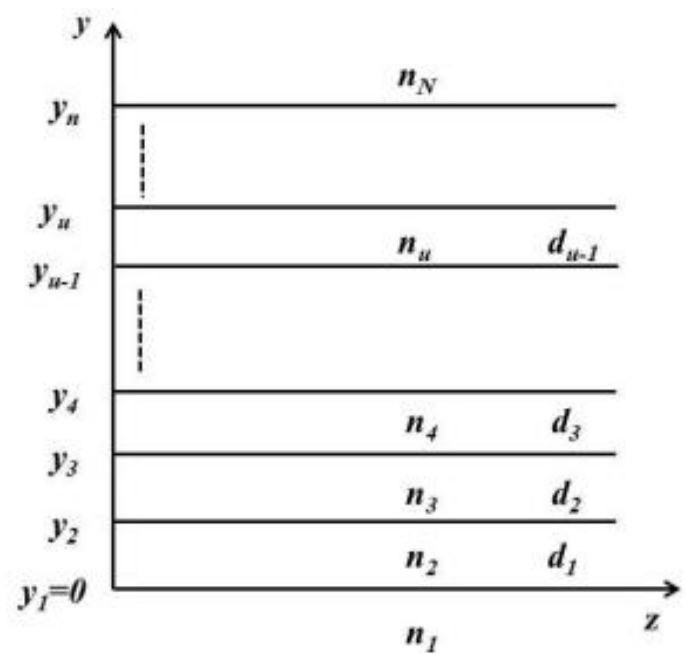

Fig.2 A multilayer planar waveguide

For the TE and TM modes of a planar waveguide the non-zero field components are $E_{x}, H_{y}, H_{z}$ and $H_{x}, E_{y}, E_{z}$ respectively. The field, $\psi$ in each layer, where $\psi$ is $E_{x}$ for TE and $H_{\mathrm{x}}$ for TM modes, can be written as $\psi(y, z, t)=\psi_{0}(y) e^{j(\omega t-\beta z)}$ and $\psi(y)$ satisfies the equation

$$
\frac{d^{2} \psi}{d y^{2}}+\left[k_{0}^{2} n^{2}(y)-\beta^{2}\right] \psi=0
$$

For a mode with the decaying solution in region 1, the field solutions can be written as

$$
\begin{aligned}
& \psi_{1}(y)=A_{1} e^{\gamma_{1} y} \quad y<0 \\
& \psi_{u}(y)=A_{u} \cos \kappa_{u}\left(y-y_{u-1}\right)+B_{u} \sin \kappa_{u}\left(y-y_{u-1}\right) \quad 1>y \geq N
\end{aligned}
$$

with, $\gamma_{1}^{2}=k_{0}^{2}\left(n_{e}^{2}-n_{1}^{2}\right), \kappa_{u}^{2}=k_{0}^{2}\left(n_{u}^{2}-n_{e}^{2}\right)$ and $n_{e}=\beta / k_{0}$ is the effective index, which is in general complex for waveguides with layers of complex refractive index. The real part of the effective index $n_{e r}$ defines the propagation constant while the attenuation coefficient is defined by the imaginary part $n_{e i}$ and is given by $\alpha=k_{0} n_{e i} \mathrm{~Np} / \mathrm{m}$.

The continuity of $\psi$ and $\frac{d \psi}{d y}$ for TE modes and $\psi$ and $\frac{1}{n^{2}} \frac{d \psi}{d y}$ for TM modes at each interface leads to the following recursion relations for the $A$ and $B$ coefficients: 


$$
\begin{gathered}
A_{2}=A_{1} ; B_{2}=\Gamma_{1} A_{1} \\
A_{u+1}=A_{u} \cos \kappa_{u} d_{u}+B_{u} \sin \kappa_{u} d_{u} \\
B_{u+1}=\Gamma_{u}\left[-A_{u} \sin \kappa_{u} d_{u}+B_{u} \cos \kappa_{u} d_{u}\right]
\end{gathered}
$$

where $\Gamma_{u}=\frac{\kappa_{u}}{\kappa_{u+1}}$ for TE modes and $\Gamma_{u}=\frac{\kappa_{u}}{\kappa_{u+1}} \frac{n_{u+1}^{2}}{n_{u}^{2}}$ for TM modes; $d_{u}=y_{u+1}-y_{u}$ is the thickness of the $u^{t h}$ layer.The field in the $\mathrm{N}^{\text {th }}$ layer can be rewritten in terms of $\kappa_{N}$ as

$$
\psi_{N}(y)=\frac{1}{2}\left[\left(A_{N}-j B_{N}\right)\left(e^{j \kappa_{N r}\left(y-y_{N-1}\right)} e^{-\kappa_{N i}\left(y-y_{N-1}\right)}\right)+\left(A_{N}+j B_{N}\right)\left(e^{-j \kappa_{N r}\left(y-y_{N-1}\right)} e^{\kappa_{N i}\left(y-y_{N-1}\right)}\right)\right]
$$

where $\kappa_{N}=\kappa_{N r}+j \kappa_{N i}$. For bound mode solutions, $\operatorname{Re}\left(n_{e}\right)>n_{N}$, in general $\kappa_{N i} \gg \kappa_{N r}$ and since only exponentially decaying fields can exist in the $\mathrm{N}^{\text {th }}$ layer, the eigenvalue equation for effective index $\left(n_{e}\right)$ of bound modes is given by

$$
F\left(n_{e}\right)=A_{N}+j B_{N}=0
$$

However, $\operatorname{Re}\left(n_{e}\right)<n_{N}, \kappa_{N r} \gg \kappa_{N i}$ and the first term of equation (4) corresponds to an incoming wave and the second term corresponds to an outgoing wave. One obtains a "leaky wave" solution by retaining only the outgoing solution. This implies that the eigenvalue equation for effective index $\left(n_{e}\right)$ of leaky modes can be written as

$$
F\left(n_{e}\right)=A_{N}-j B_{N}=0
$$

Further, as pointed out by some early authors [9,10], even for $\operatorname{Re}\left(n_{e}\right)>n_{N}$, one can obtain a 'leaky mode' solution of the boundary value problem. In equation (4), the first term represents an incident wave and the second term an outgoing wave due to the relatively small $\kappa_{N r}$ term. Hence a leaky mode solution will be obtained with the effective index of the leaky mode is obtained by the eigen value equation

$$
F\left(n_{e}\right)=A_{N}-j B_{N}=0
$$

The dielectric-metal-dielectric (DMD) plasmonic waveguide configurationshown in Figs 1(b) and (c), the layer, $m=2$ is a gold metal layer of thickness $d$ with $\varepsilon_{2}=-\varepsilon_{2 r}-j \varepsilon_{2 i}$, forms a three layer dielectric-metal-dielectric waveguide structure $(\mathrm{N}=3)$. The bound and leaky modes supported by such waveguides and their applications will be discussed in the next sections.

\section{THE DIELECTRIC-METAL-DIELECTRIC WAVEGUIDE}

Before discussing the modes of the DMD waveguide it is also important to understand the TM surface plasmon mode supported by a metal-dielectric interface [Fig.1 (a)]. The fields for a bound TM mode on the interface, $y=0$, can be written as

$$
\begin{gathered}
H_{x 1}(y)=A_{1} e^{\gamma_{1} y} y>0 \\
H_{x 2}(y)=A_{2} e^{-\gamma_{2} y} \quad y<0
\end{gathered}
$$

where $\gamma_{1}^{2}=k_{0}^{2}\left(n_{e}^{2}-\varepsilon_{d}\right)$ and $\gamma_{2}^{2}=k_{0}^{2}\left(n_{e}^{2}-\varepsilon_{m}\right), \varepsilon_{d}=n_{d}^{2}$ and $\varepsilon_{m}=n_{m}^{2}$ are the dielectric constants of the dielectric and metal layer respectively. The continuity of $H_{x}$ and $\frac{1}{n^{2}} \frac{d H_{x}}{d y}$ leads to the expression for the effective index, $n_{S P}=n_{S P r}-j n_{S P i}$ of the surface plasmon mode.

$$
n_{S P}=\left(\frac{\varepsilon_{m} \varepsilon_{d}}{\varepsilon_{m}+\varepsilon_{d}}\right)^{1 / 2}
$$

Fig. 3 shows the variation of $n_{S P}$ of the surface plasmon mode withdielectric of refractive index $n_{d}$ for gold with $\varepsilon_{\mathrm{m}}=(-44.9512-\mathrm{j} 5.9417)$ at $\lambda_{0}=1.15 \mu \mathrm{m}$. On increasing the refractive index $n_{d}$, thereal part of effective index of the surface plasmon mode keeps on increasing.In order to study the various TM surface modes supported by the dielectric-metal-dielectric waveguide, we considered a gold $(A u)$ metal layer, with dielectric $\operatorname{constant} \varepsilon_{2}=(-$ 
$44.9512-\mathrm{j} 5.9417)$ at $\lambda_{0}=1.15 \mu \mathrm{m}$ of thickness $d=50 \mathrm{~nm}$ sandwiched between the semi-infinite dielectric layers of refractive indices $n_{1}$ and $n_{3}$. To study the effect of the index contrast between the dielectrics on modes supported by such a waveguide with dielectric layers of relatively high dielectric constant, we used $n_{3}^{2}=9.5$ and varied $n_{1}$. Such a DMD waveguide can, in general, support four distinct modes: two bound modes, namely, the symmetric bound $\left(s_{b}\right)$ and the antisymmetric bound $\left(a_{b}\right)$ modes and two leaky modes, namely, the symmetric leaky $\left(s_{l}\right)$ and the antisymmetric leaky $\left(a_{l}\right)$ modes.In Fig. 4 , the effective index of these modes are plotted as a function of refractive index, $n_{1}$.Fig. 4 also shows the effective indices of the surface plasmon mode supported by the $n_{1}-A$ uand $n_{3}-A$ uinterfaces and the refractive indices $n_{1}$ and $n_{3}$. From these calculations the following significant conclusions can be drawn for various modes supported by the DMD waveguide.
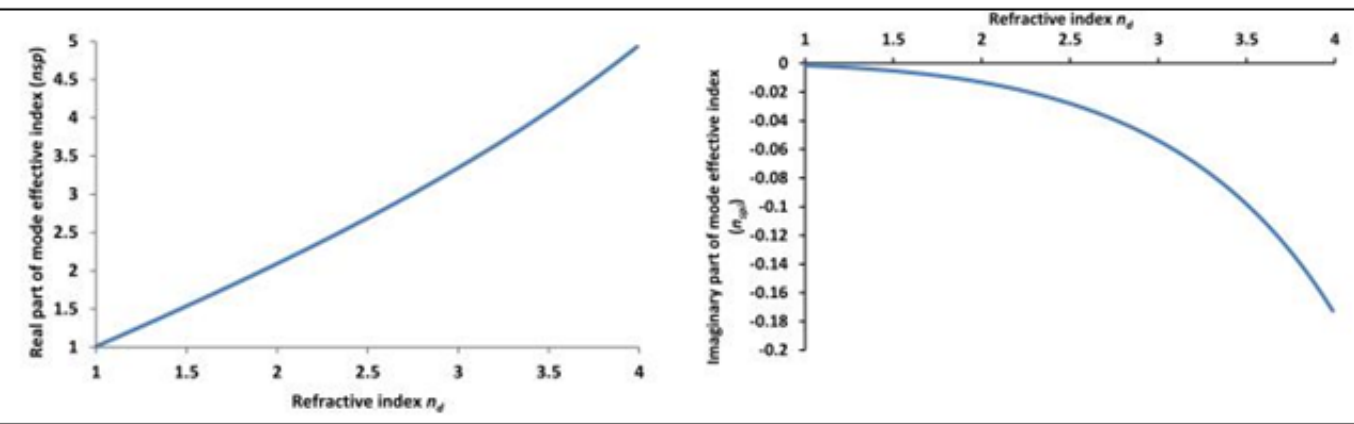

Fig.3Variation of (a) real part of mode effective index, (b) Imaginary part of effective index of the surface plasmon modeas a function of refractive index, $n_{d}$, of dielectric layer.
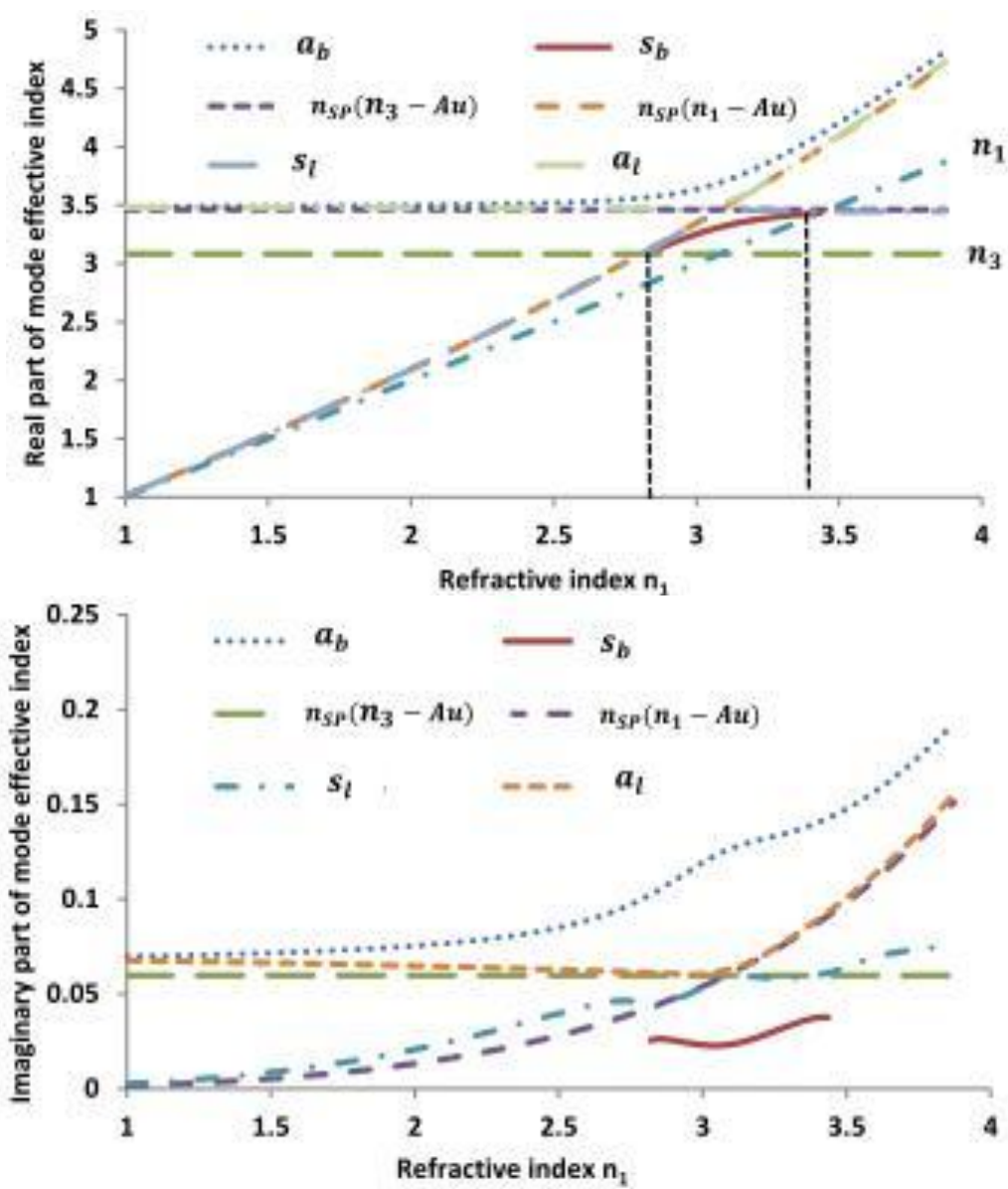

Fig. 4 (a) Real part and (b) Imaginary part of the effective index of the modes supported by the three layer DMD waveguide plotted as a function of $n_{1}$ for $\varepsilon_{m}=(-44.9512-\mathrm{j} 5.9417), n_{3}=3.0822$ and $d=50 \mathrm{~nm}$. 
3.1. Bound Modes

3.1.1. The antisymmetric bound $\left(a_{b}\right)$ mode

The antisymmetric bound $\left(a_{b}\right)$ mode exists for all contrast in refractive indices of the dielectric layers. The real part of the effective index for the antisymmetric bound $\left(a_{b}\right)$ mode is always greater than the effective index of the surface plasmon mode supported by the high index dielectric and metal interface. For $n_{3}(3.0822)$ $>n_{1}$, the real part of the effective index for $a_{b}$ mode for high contrast in indices of the dielectric layers decreases to the effective index of the surface plasmon mode for the semi-infinite high index and metal layer, i.e., $n_{S P}\left(n_{3}-A u\right)$. For $n_{1}>n_{3}$, the effective index for the $a_{b}$ mode again as contrast increases tends toward the effective index of the surface plasmon (SP) mode supported by the interface between the high index layer and metal layer, i.e., $n_{S P}\left(n_{1}-A u\right)$. The $a_{b}$ mode exhibits high loss as seen by the imaginary part of the effective index plotted in Fig. 4(b) and hence is referred to as short range surface plasmon (SRSP) mode. In the region of low contrast between $n_{1}$ and $n_{3}$ marked by the dashed vertical lines, both the antisymmetric and symmetric bound modes exist.Fig.5 shows the variation of the effective indices in this region with thickness, $d$, of the metal layer. The effective index of these modes is a strong function of metal thickness in the low contrast region and hence this can be tuned by the change in metal thickness. This property has been used in the early polarizer designs [5]. It is this mode which also plays a vital role in the recently designed polarizers for silicon on insulator (SOI) waveguide [13]. The Fig.6shows the modal field profiles of the bound modes of the dielectricmetal-dielectric waveguide for $n_{3}=3.0822$ and the other parameters as $\varepsilon_{2}=(-44.9512-\mathrm{j} 5.9417)$ with the thickness $d=50 \mathrm{~nm}$ at $\lambda_{0}=1.15 \mu \mathrm{m}$ for three different values of $n_{1}$. Fig.6(a) and 6(c) show that for the antisymmetric bound mode the field has peaks on the interface and decaying nature in the dielectric regions. For $n_{1} \neq n_{3}$, the fieldis higher at the high index layer and metal interface while for $n_{1}=n_{3}$, the field peaks are equal at both interfaces shown in Fig. 6(b). These are essentially the antisymmetric mode formed by coupling between the surface plasmon modes at the two interfaces.

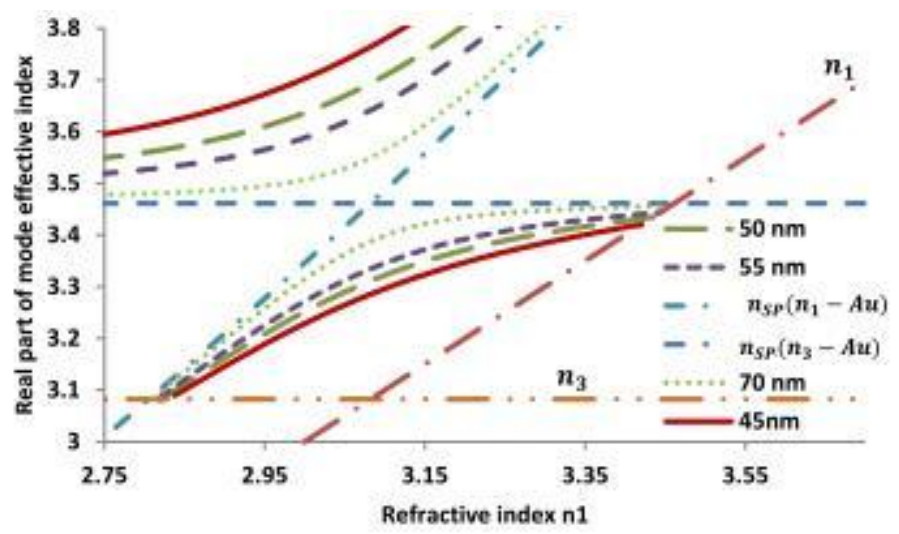

Fig.5 Real part of the effective index of the symmetric and antisymmetric bound mode for $n_{3}=3.0822$, $\varepsilon_{m}=(-44.9512-\mathrm{j} 5.9417)$ at varying thickness $d=45,50,55$ and $70 \mathrm{~nm}$.

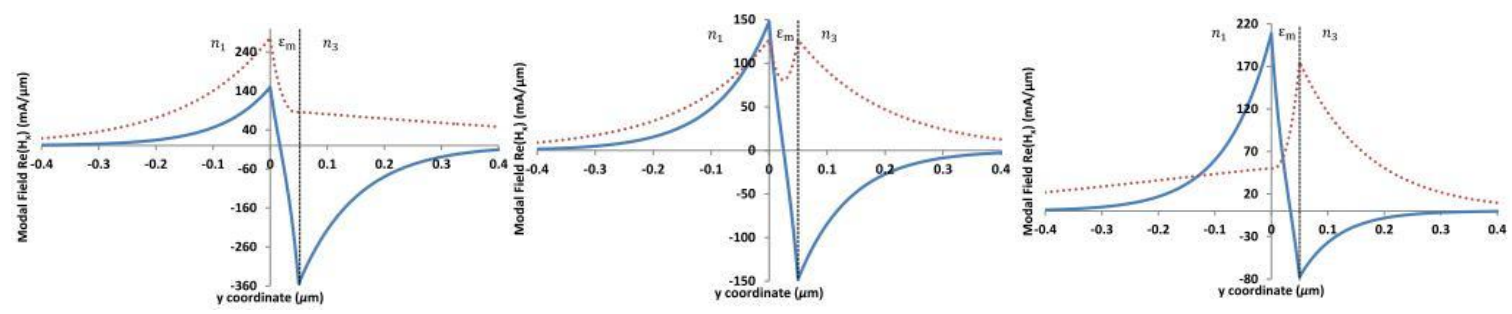

Fig.6 Modal filed profile of theantisymmetric bound mode (solid line) and symmetric bound mode(dotted line) for $\varepsilon_{m}=(-44.9512-\mathrm{j} 5.9417), n_{3}=3.0822$ and $\mathrm{d}=50 \mathrm{~nm}$ at $\lambda_{0}=1.15 \mu \mathrm{m}$ for(a) $n_{1}=2.8284$ (b) $n_{1}=$ 3.0822 (c) $n_{1}=3.4351$

\subsubsection{The symmetric bound $\left(s_{b}\right)$ mode}

The symmetric bound $\left(s_{b}\right)$ mode exists only in the low contrast regions. For $n_{3}>n_{1}$ the real part of the effective index of the $s_{b}$ mode increases as $n_{1}$ increase to a maximum value at $n_{1}=n_{3}$ and for $n_{3}<n_{1}$ it again decreases. The $s_{b}$ mode exists as long as its effective index is higher than the refractive index of both the dielectric layers. It may be noted that the effective index of $s_{b}$ mode is always less than the effective index of 
surface plasmon mode of the low index and metal interface as shown with clarity in Fig.5. The effective index and cut-off index $n_{1}$ depend on the metal film thickness. In regions when the effective index of the $s_{b}$ mode is lower than that of the high index layer, it transforms to a leaky mode discussed in the next section. For $n_{1}>n_{3}$, the $s_{b}$ mode can exist only when its effective index is below the refractive index of the high index layer $n_{1}$ and its value is always less than that of the SP mode of the $n_{S P}\left(n_{3}-A u\right)$ interface. The attenuation of these modes is lower than that of the antisymmetric modes and hence these modes are referred as the long range surface plasmon (LRSP) modes. The $s_{b}$ mode exists only when the following condition satisfied:

$$
\frac{\varepsilon_{h}-\varepsilon_{l}}{\varepsilon_{h} \varepsilon_{l}}<\frac{1}{\left|\varepsilon_{m}\right|}
$$

where $\varepsilon_{h}=n_{h}^{2}$ is high dielectric constant, $\varepsilon_{l}=n_{l}^{2}$ is low dielectric constant and $\varepsilon_{m}$ is the dielectric constant of the metal layer. As this condition is not dependent on the metal thickness it will only state whether the $s_{b}$ mode can exist or not, but may not define the actual cut-off. The Fig.6shows that for the symmetric bound modes the modal field has peaks at the interfaces and decays in the dielectric regions.For $n_{1} \neq n_{3}$, the field is higher at the low index layer and metal interface while for $n_{1}=n_{3}$, the field peaks are equal at both interfaces.
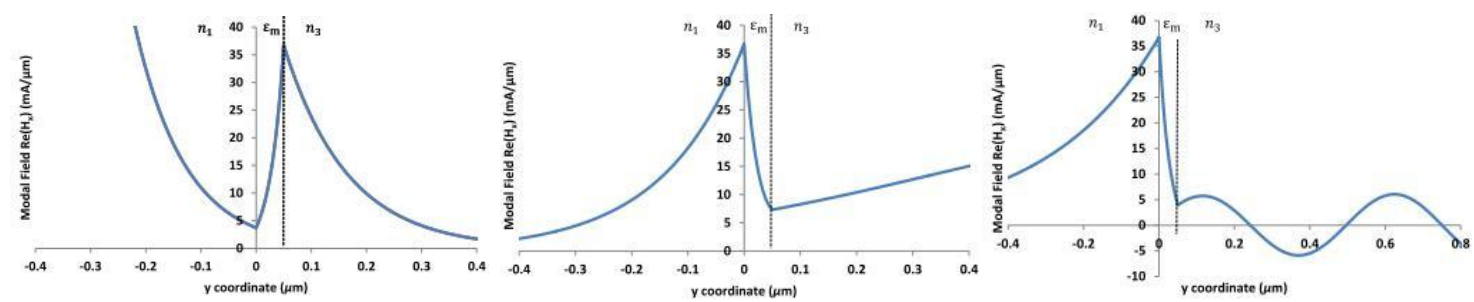

Fig. 7 Modal filed profile a) Antisymmetric leaky mode $\left(n_{e}=(3.466-\mathrm{j} 0.0611)\right.$, b) Symmetric leaky mode $\left(n_{e}=(3.102-\mathrm{j} 0.0433)\right.$, for $n_{1}=2.8284, \varepsilon_{m}=(-44.9512-\mathrm{j} 5.9417), n_{3}=3.0822$ and $=50 \mathrm{~nm}(\mathrm{c})$

Symmetric leaky mode $n_{e}=(2.093-\mathrm{j} 0.0206)$ below cutoff point for $n_{1}=2.0$

\subsection{Leaky modes}

3.2.1.Theantisymmetric leaky $\left(a_{l}\right)$ mode

The antisymmetric leaky $\left(a_{l}\right)$ mode exists for all index contrast between the dielectric layers and is localized at the interface of the high index layer and leaks out into the low index layer. The effective index of the $a_{l}$ mode converges to that of the SP mode at the high index-metal interface, i.e., $n_{S P}\left(n_{3}-A u\right)$ interface for $n_{3}>n_{1}$ and $n_{S P}\left(n_{1}-A u\right)$ interface forn $n_{1}>n_{3}$ for large metal thicknesses. The field for the antisymmetric leaky $\left(a_{l}\right)$ mode plotted in Fig.7(a) shows a peak on the high index-metal interface decaying exponentially in the high index region but grows exponentially into the low index dielectric medium in contact with the other interface.Hence this mode does not have any physical significance.

3.2.2.The symmetric leaky $\left(s_{l}\right)$ mode

The symmetric leaky $\left(s_{l}\right)$ mode also exists for all index contrast between the dielectric layers. For bound mode region, $n_{1}<\operatorname{Re}\left(n_{e}\right)>n_{3}$, the fields in the region of higher index $\left(n_{3}\right)$ dielectric layer is exponentially increasing as shown in Fig.7(b). The symmetric leaky $\left(s_{l}\right)$ mode is localized at the interface of the low index layer and leaks out in high index layer. The effective index of the $s_{l}$ mode converges to the surface plasmon mode $n_{S P}\left(n_{1}-A u\right)$ for $n_{3}>n_{1}$ and similarly has all the characteristics of a surface plasmon moden $n_{S P}\left(n_{3}-A u\right)$ for $n_{1}>n_{3}$ for large metal thickness. Hence this mode also does not have any physical significance.

However, for $n_{1}<\operatorname{Re}\left(n_{e}\right)<n_{3}$ the field in region 3 is oscillatory gives the leaky mode solution defined by equation (6). Such a solution is bound at the lower interface i.e., $n_{1}-n_{m}$, but power leaks into the higher index region, $n_{3}$. This mode is excited in the Kretschmann configuration used for sensor applications discussed in section 4.1.At high contrast dielectric layers, below the cutoff, the symmetric bound mode no longer exist and lead to the symmetric leaky mode with effective index lower than the high index layer of the waveguide. The Fig.7(c) is the modal field profile of the leaky mode below the cutoff domain of symmetric bound mode for $n_{1}=2.0$ and keeping remaining parameters the same.

\subsection{Effect of metal thickness}

To study the effect of metal thickness, we considered the three layer asymmetric structure with low contrast in refractive index of the dielectric layers, i.e., $n_{1}=2.9154, n_{3}=3.0822$ and $\varepsilon_{2}=(-44.9512-$ j5.9417) at $\lambda_{0}=1.15 \mu \mathrm{m}$ with varying metal layer thickness $d$ as shown in Fig.8(a). This configuration supports two bound modes and two leaky modes. The effective index of the $a_{b}$ mode decreases on increasing the metal layer 
thickness and converges to the effective index of the surface plasmon mode of the $n_{3}-A u$ interface. In contrast, the effective index of the $s_{b}$ mode increases on increasing the metal layer thickness and converge to the effective index of the surface plasmon mode for the $n_{1}-A u$ interface. Below the cutoff thickness the $s_{b}$ mode ceases to exist as a guided mode and re-appears as the symmetric leaky mode for a lower metal thickness with effective index $n_{e}$ (leaky mode) $<n_{3}$. The modal loss for the long range $s_{b}$ mode is less than the short range $a_{b}$ mode. The loss values approach that of the surface mode supported by a single metal-dielectric interface when the metal thickness is large as shown in Fig.8(b). When the $s_{b}$ mode reappers as the leaky mode, the attenuation also shows a sudden increase.As mentioned, it is this mode which is excited in most sensor applications.

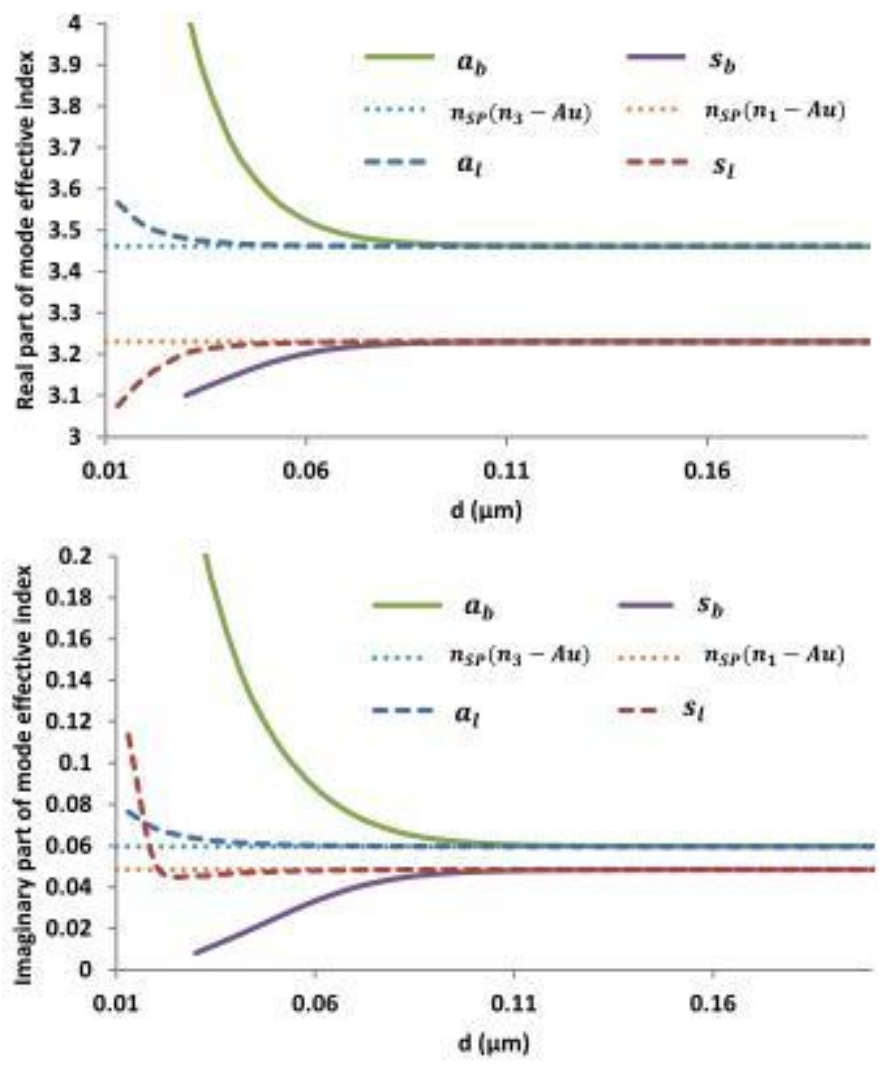

Fig.8(a) Real part and (b) Imaginary part of the mode index of the three layer structure is plotted as a function of thickness $d$ of metal layer for $\varepsilon_{m}=(-44.9512-\mathrm{j} 5.9417), n_{3}=3.0822, n_{1}=2.9154$

\section{APPLICATIONS OF THE VARIOUS MODES SUPPORTED BY DMD WAVEGUIDES}

\subsection{In Surface Plasmon Resonance (SPR) Sensors}

As seen in Section 3, the propagation constant of the surface plasmon mode supported by a metal dielectric interface is a sensitive function of the refractive index of the dielectric layer. This property along with the use of dielectric layers in which the refractive index changes due to the chemical or biological interactions resulted in the development of various gas, chemical and bio-molecule sensors. They are based on resonant excitation of the surface plasmon mode and are known as surface plasmon resonance (SPR) sensors.

The monochromatic p-polarized light is incident through the prism on the metal surface and the reflected light measured as a function of angle of incidence. The most popular Kretschmann configuration which uses attenuated total reflection (ATR) for the excitation of surface plasmon mode for sensor applications is shown in Fig. 9(a). In this configuration, a thin metal film $\left(\varepsilon_{m}\right)$ is deposited on the surface of the glass prism $\left(n_{3}\right)$ is immersed into the analyte to be sensed. It is widely agreed that the reflection dip occurs at an angle of incidence where $n_{3} \sin \theta$ equals the effective index of the surface plasmon wave supported by the metal-analyte interface i.e., $n_{3} \sin \theta=\operatorname{Re}\left(\sqrt{\frac{n_{1}^{2} \varepsilon_{m}}{n_{1}^{2}+\varepsilon_{m}}}\right)$. The SPR curves of the type shown in Fig. 9(b) for different indices $n_{1}$ are obtained. Hence, the angle at which theminima occurs is a measure of the refractive index of the analyte layer. These curves correspond to a typical configuration of a fused glass prism of refractive index, $n_{3}=1.45$ with a $50 \mathrm{~nm}$ metal layer of gold $\left[\varepsilon_{2}=(13.0822-\mathrm{j} 0.9977)\right]$ at $\lambda_{0}=0.633 \mu \mathrm{m}$. The surface plasmon mode excited hence corresponds to that of the DMD waveguide silica-Au-analyte. With the analyteof refractive index in the range $n_{1}=$ 1.0 and 1.33, as most of the sensing material will be in gas (air) or liquid (water) environment,the effective indices of the antisymmetric $\left(a_{b}\right.$ and $\left.a_{l}\right)$ modes are always greater than the effective index of thesurface plasmon

DOI: 10.9790/2834-1202010819 $\quad$ www.iosrjournals.org $14 \mid$ Page


mode of the high index layer and metal interface, i.e., $n_{S P}\left(n_{3}-A u\right)=(1.5817-\mathrm{j} 0.0114)$, and hence cannot be excited by a prism of refractive index 1.45.The DMD waveguide will not support the symmetric bound $\left(s_{b}\right)$ mode because the effective index of the SP mode of the metal-air interface and metal-water interface obtained as $n_{S P}\left(n_{1}-A u\right)=(1.0411-\mathrm{j} 0.0068)$ and $\left.n_{S P}\left(n_{1}-A u\right)=(1.4240-\mathrm{j} 0.0122)\right]$ respectively are lower than the refractive index of high index layer, $n_{3}=1.45$ as discussed explicitly in section 3.1.2. The only mode supported is the symmetric leaky $\left(s_{l}\right)$ mode below the cutoff of the $s_{b}$ mode and which haseffective index less than 1.45 for the range of analyte refractive indices considered and the resonant dip in the SPR corresponds to the excitation of this mode.
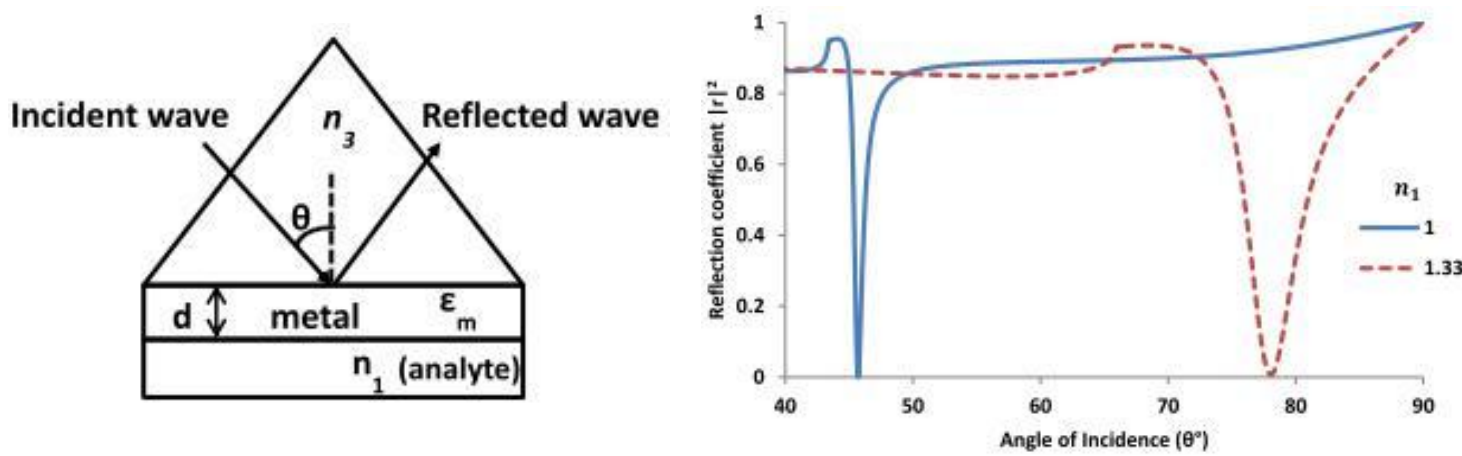

Fig.9The Kretschmann configuration for excitation of surface mode. (b) Surface plasmon resonance curves for $n_{3}=1.45, \varepsilon_{m}=(-13.0822-\mathrm{j} 0.9977), n_{1}=1.0$ and $1.33, d=50 \mathrm{~nm}$ at $\lambda_{0}=0.633 \mu \mathrm{m}$.
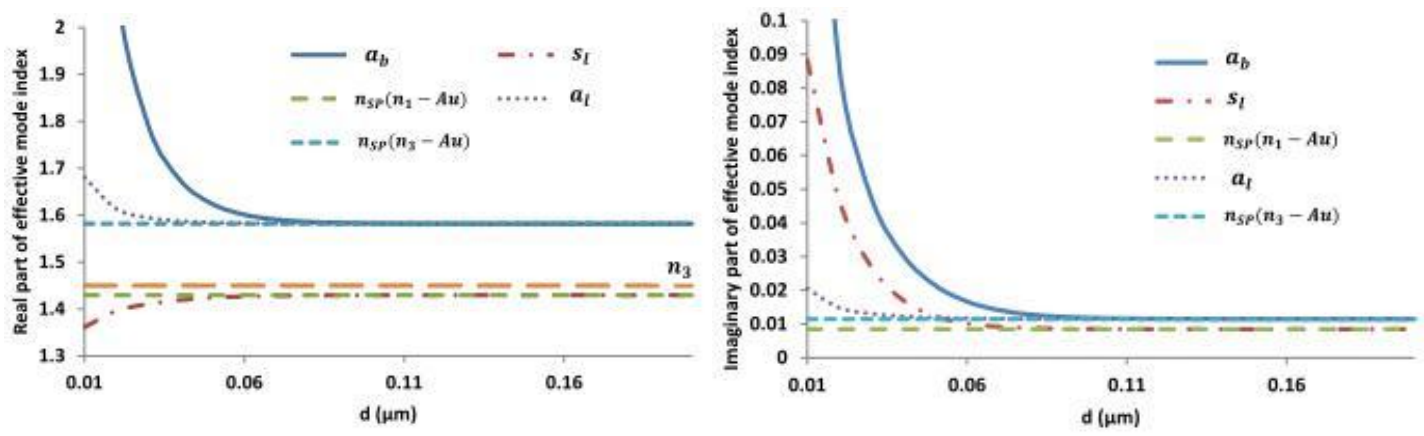

Fig.10 (a) Real part and (b) Imaginary part of the mode effective index of the threelayer structure is plotted as a function of thickness $d$ of metal layer for $\varepsilon_{m}=(-13.0822-\mathrm{j} 0.9977), n_{3}=1.45, n_{1}=1.33$ at $\lambda_{0}=0.633 \mu \mathrm{m}$.

The effective indices of the modes supported by this DMD waveguide are plotted as a function of metal thickness for refractive indices, $n_{1}=1.33, n_{3}=1.45$ and $\varepsilon_{2}=(13.0822-\mathrm{j} 0.9977)$ at $\lambda_{0}=0.633 \mu \mathrm{min}$ Fig. 10. This configurationsupports one bound mode and two leaky modes. The effective index of the $a_{b}$ mode decreases on increasing the metal layer thickness and converges to the effective index of thesurface plasmon mode of the $n_{S P}\left(n_{3}-A u\right)$ interface.The $s_{b}$ mode will exist only if eq.(10) satisfies. For the above parameters the left hand side is 0.089 and right hand side is equal to 0.076 and we can conclude that the $s_{b}$ mode cannot exist. The only mode which exists and is excited by the evanescent field of the ATR is the symmetric leaky $s_{l}$ mode. The real part of the effective index of the $s_{l}$ mode increases with metal thickness and for thickness $\sim 60 \mathrm{~nm}$ converges $\operatorname{ton}_{S P}\left(n_{3}-A u\right)$.The modal field profile of the symmetric leaky mode for $n_{1}=1.0$ is shown in Fig.11. The field is localized at the $n_{1}-A u$ interface and shows the oscillatory behavior in the high index layer $\left(n_{3}\right)$. By varying the refractive index of the analyte $\left(n_{1}\right)$, the field localization at the metal-dielectric (low index layer) changes and hence used for sensor applications. 


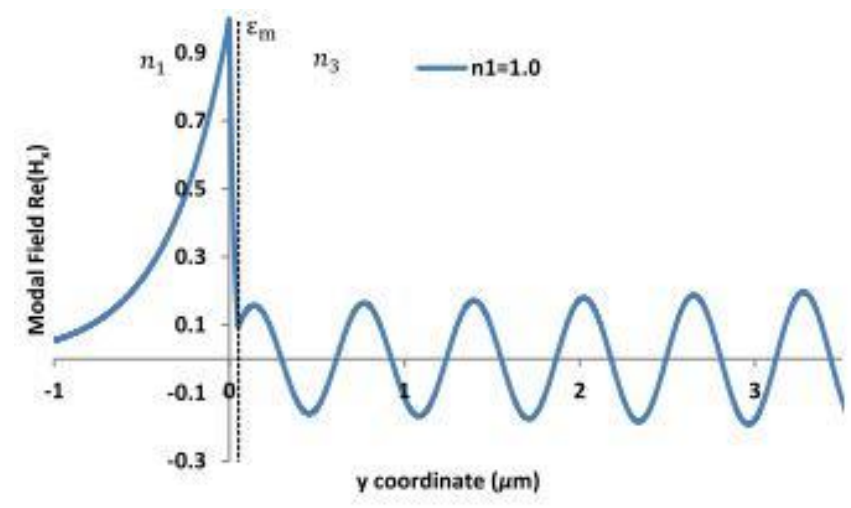

Fig.11 (a) Modal field profile of the leaky surface plasmon mode with effective index $\left(n_{e}=1.0411-\mathrm{j} 0.0068\right)$ for $n_{1}=1.0$ for the Kretschmann configuration.

\subsection{In Waveguide Polarizers}

Another important application of the surface plasmon mode of DMD waveguides is in TE/TM waveguide polarizers. A metal-cladplasmonic waveguide can be designed to act as a polarizer in integrated optical circuits $[3-6,12,13]$. The metallic layer provides polarization discrimination because losses for TM modes were higher than losses for TE polarized modes due to penetration of the TM fields into the metal. However, the design for such polarizers has been usually based on parametric simulations. Now with an understanding of surface plasmon modes supported by a metal dielectric interface these polarizers can be understood in terms of coupling between the guided TM mode of the dielectric waveguide and the lossy surface plasmon TM mode of a DMD waveguide.Chen and Wang [12] have reported a polarizer in low index silica dielectric waveguide with aluminum as a metal layer sandwiched in-between $n_{3}$ (buffer) layer and $n_{1}$ (cover) layer shown in Fig.12 with corresponding refractive indices. The single moded dielectric waveguide is formed by the substrate-core-buffer.

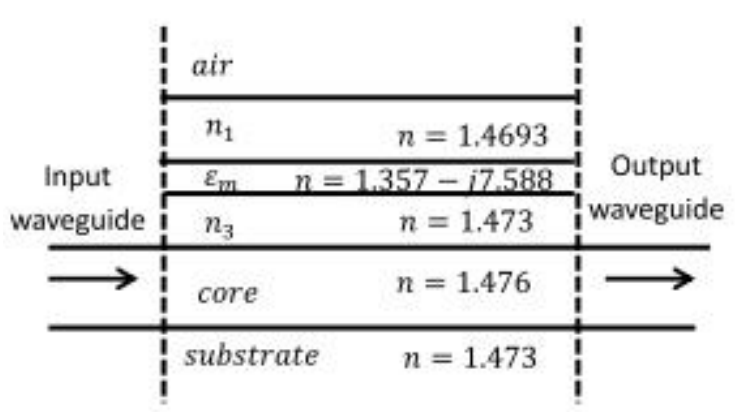

Fig.12Surfaceplasmon waveguide polarizer in silica waveguide

The DMD $\left(n_{1} / \varepsilon_{m} / n_{3}\right)$ waveguide has a low contrast in refractive indices of the dielectric layersand hence supports all four modes namely antisymmetric bound mode $\left(a_{b}\right)$, antisymmetric leaky mode $\left(a_{l}\right)$, symmetric bound mode $\left(s_{b}\right)$ and symmetric leaky mode $\left(s_{l}\right)$. The effective indices of the $a_{b}$ and $s_{b}$ modes as a function of thickness of the metal layer along with the effective index of the TM guided mode of the dielectric waveguide are shown in Fig. 13. The TM guided mode of the dielectric waveguide (substrate/core $/ n_{3}$ ) is phase matched, i.e., has the same effective index, with the $s_{b}$ mode of the DMD waveguide at $d=9.6 \mathrm{~nm}$. 


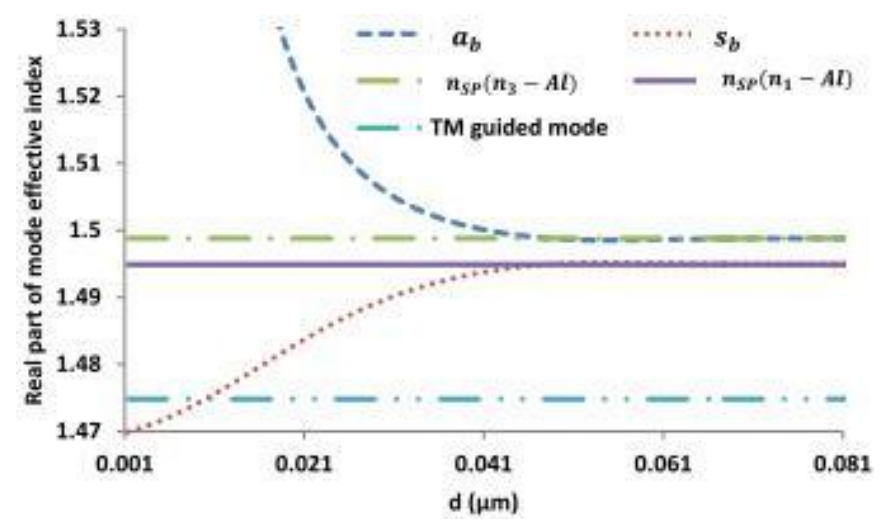

Fig. 13 Real part of the $a_{b}$ ands $s_{b}$ mode effective index of the DMD waveguide is plotted as a function of thickness $d$ of metal layer for $n_{1}=1.4693, n_{3}=1.473$ at $\lambda_{0}=0.633 \mu \mathrm{m}$. The TM guided mode is plotted for core thickness of $3 \mu \mathrm{m}$.
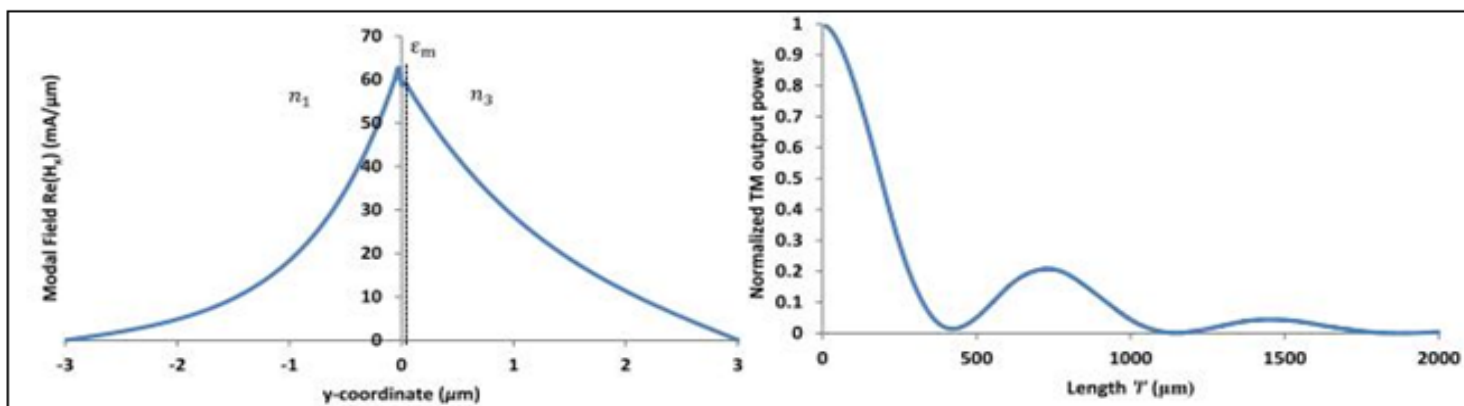

Fig.14(a) Modal field profile for symmetric bound mode with effective index $\left(n_{e}=1.4748-\mathrm{j} 0.0002\right)$ (b) Variation of normalized power in the output waveguide with length of the polarizer sectionfor $n_{1}=1.4693$, $n_{3}=1.473$ and $d=9.6 \mathrm{~nm}$, buffer layer thickness of $2 \mu \mathrm{m}$, core layer thickness $3 \mu \mathrm{m}$, cover layer $\left(n_{1}\right)$ thickness of $2 \mu \mathrm{m}$ at $\lambda_{0}=0.633 \mu \mathrm{m}$

Hence, for metal thickness $d=9.6 \mathrm{~nm}$, the two waveguides are synchronous and power will be completely coupled out of the dielectric waveguide into the lossy DMD waveguide at coupling length. The variation of the TM mode power coupled to the output waveguide is shown in Fig. 14(b). More recently a TE pass polarizer has been reported for silicon on insulator (SOI) waveguide with a gold metal layer and an additional buffer layer with corresponding refractive indices shown in Fig. 15. The dielectric waveguide is the $\mathrm{SiO}_{2}-\mathrm{Si}-\mathrm{SiO}_{2}-\mathrm{Si}_{3} \mathrm{~N}_{4}$ waveguide and the DMD waveguide is $\mathrm{SiO}_{2}-\mathrm{Si}_{3} \mathrm{~N}_{4}-\mathrm{Au}-\mathrm{Air}$. In this case the DMD waveguide has a high contrast in the refractive indices of the dielectric layers and hence can support only the antisymmetric modes and the symmetric leaky mode.

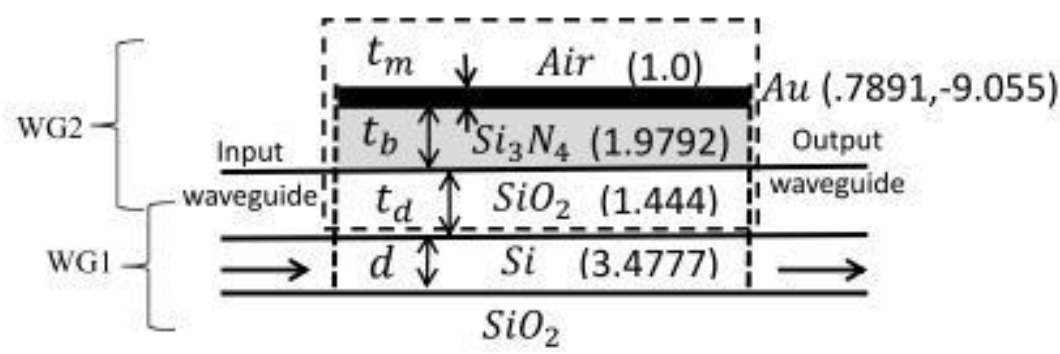

Fig.15Surface plasmon waveguide polarizer in Silicon on Insulator waveguide.

The effective index of the antisymmetric bound mode is plotted as a function of buffer layer thickness $t_{b}$ with $t_{m}=50 \mathrm{~nm}$ along with the effective index of the TM guided mode of the dielectric waveguide in Fig. 16.The TM guided mode of the dielectric waveguide is phase matched with the antisymmetric bound mode of the DMD waveguide at $t_{b}=500 \mathrm{~nm}$. 

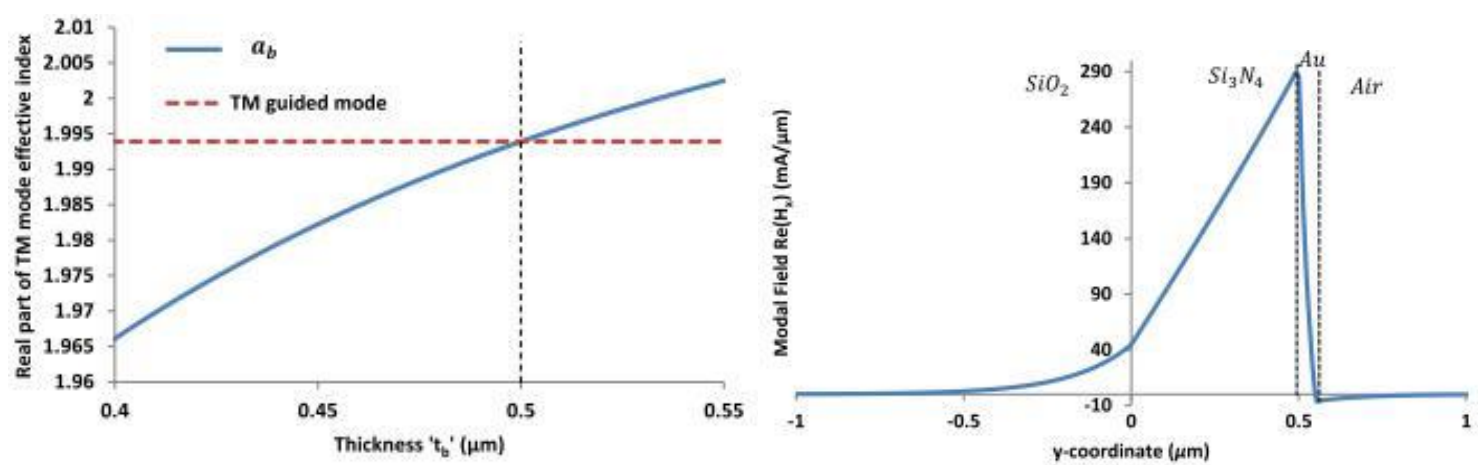

Fig.16(a) Real part of the $\boldsymbol{a}_{\boldsymbol{b}}$ mode effective index of the DMD waveguide plotted as a function of thickness $\boldsymbol{t}_{\boldsymbol{b}}$ at $\boldsymbol{t}_{\boldsymbol{m}}=50$ nmfor $\boldsymbol{\lambda}_{0}=1.55 \mu \mathrm{m}$ along with the TM guided mode of the dielectric waveguide for $\boldsymbol{d}=210.94 \mathrm{~nm}, \boldsymbol{t}_{\boldsymbol{d}}=300 \mathrm{~nm}$.(b) Modal field profile of the $\boldsymbol{a}_{\boldsymbol{b}}$ bound mode of the DMD waveguide at phase matching thickness $\boldsymbol{t}_{\boldsymbol{b}}=500 \mathrm{~nm}$.

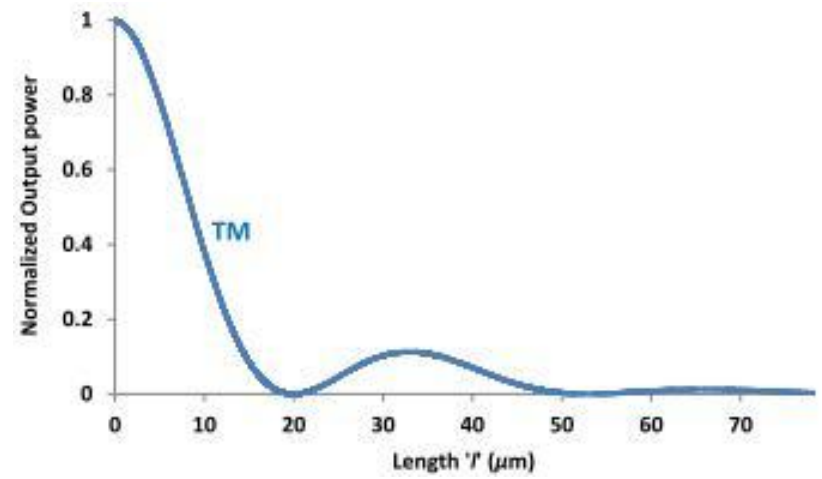

Fig.17Variation of normalized power in the output waveguide with length of the polarizer sectionford $=210.94 \mathrm{~nm}, \boldsymbol{t}_{\boldsymbol{d}}=300 \mathrm{~nm}, \boldsymbol{t}_{\boldsymbol{b}}=500 \mathrm{~nm}$ and $\boldsymbol{t}_{\boldsymbol{m}}=50 \mathrm{~nm}$.

Hence, where the polarizer section is excited by a TM mode the total field is confined in the guiding layer of the dielectric waveguide at the input end and as it propagates the power completely out couples periodically to the metal interface of the DMD waveguide. Normalized power of the TM mode in the output waveguide as a function of polarizer section length is plotted in Fig.17. At a length of about $20 \mu \mathrm{m}$ no power is obtained in the TM mode of the output waveguide.

\section{CONCLUSION}

In conclusion, we have carried out a detailed study of the modes supported by the dielectric-metaldielectric waveguide. Our results show that although in general two bound modes can be supported by such waveguides. The low loss symmetric bound mode is supported only when the index contrast between the dielectrics is low and branches to a leaky mode at high contrast. For the long range surface plasmon bound mode to exist the condition, $\frac{\varepsilon_{h}-\varepsilon_{l}}{\varepsilon_{h} \varepsilon_{l}}<\frac{1}{\left|\varepsilon_{m}\right|}$ must be satisfied, where $\varepsilon_{h}$ is high dielectric constant, $\varepsilon_{l}$ is low dielectric constant and $\varepsilon_{\mathrm{m}}$ is the metal dielectric constant. We have also detailed some applications of the DMD waveguides with an emphasis on identifying the participating mode in each application.

\section{ACKNOWLEDGEMENTS}

The authors would like to thank University of Delhi Research and Development Grants (India) for the financial support.

\section{REFERENCES}

[1] J. M. Pitarke, V. M. Silkin, E. V. Chulkov, and P. M. Echenique, Theory of surface and surface-plasmon polaritrons, Rep. Prog. Phys. 70, 2007, 1-87

[2] J. R. Sambles, G. W. Bradbery anf F. Yang, Optical excitation of surface plasmons: an introduction, Contemp. Phys., 32( 3), 1991, $173-183$

[3] J. N. Polky and G. L. Mitchell, Metal-clad planar dielectric waveguide for integrated optics, J. of the Optical Society of America, 64(3), 1974, 274-279.

[4] Y.Yamamoto, T.Kamiya and H.Yanai, Characteristics of optical guided modes in multilayer metal-clad planar optical guide with low-index dielectric buffer layer, J. of the Optical Society of America, 11(9), 1975, 729-736.

[5] K. H. Rollke and W. Sohler, Metal-clad waveguide as cutoff polarizer for integrated optics, J. of Quantum Electronics, 13(4), 1977, 141-145. 
[6] I. Avrutsky, Integrated optical polarizer for silicon-on-insulator waveguides using evanescent wave coupling to gap plasmonpolaritrons, J. of Quantum Electronics, 14( 6), 2008, 1509-1514.

[7] W. Johnstone, G. Stewart, T. Hart and B. Culshaw, "Surface Plasmon Polaritons in the Thin Metal Films and their Role in Fiber Optic Polarizing Devices", Journal of Lightwave Technology, 8(4), 1990, 538-544.

[8] A. Baliyan, P. Bhatia, B. D. Gupta, E. K. Sharma, A. Kumari, R. Gupta, Surface plasmon resonance based fiber optic sensor for the detection of triacylglycerides using gel entrapment technique,Sensors and Actuators B chemical, 188, 2013, 917-922.

[9] J. J. Burke and G. I. Stegeman, Surface-polariton-like waves guided by thin, lossy metal films, Phyical review B, 33(8), 1986, 51865201.

[10] M. N. Zervas, Surface plasmon-polaritron waves guided by thin metal films, Optics letters, 16(10), 1991, $720-722$.

[11] E. K. Sharma and M. P. Singh, Multilayer waveguide devices with absorbing layers: an exact analysis,J. Opt. Commun., 14(4), 1993, 134-137.

[12] C.H. Chen and L. Wang, Maximization of Extinction Ratios of Thin-Metal-Clad Optical Waveguide Polarizers with Proper Dielectric-Cover-Layer Thickness, Jpn. J. Appl. Phys., 39,2000, 4130-4137.

[13] S. Sital and E. K. Sharma, Design Methodology for TE-Pass Polarizer in SOI Waveguides, Journal of Opt Quant Electron, 48, $2016,369$. 